\title{
Deciphering the Role of Physical Activity in Stress Management During A Global Pandemic in Older Adult Populations: A Systematic Review Protocol
}

Ryan Churchill ( $\square$ ryan_churchill@sfu.ca)

Simon Fraser University https://orcid.org/0000-0001-6510-4810

Indira Riadi

Simon Fraser University

Lucy Kervin

Simon Fraser University

Kelly Teo

Simon Fraser University

Theodore Cosco

Simon Fraser University

\section{Protocol}

Keywords: Stress management, physical activity, pandemic, intervention, older adults, systematic review

Posted Date: August 19th, 2020

DOl: https://doi.org/10.21203/rs.3.rs-59516/v1

License: (c) (i) This work is licensed under a Creative Commons Attribution 4.0 International License.

Read Full License

Version of Record: A version of this preprint was published at Systematic Reviews on May 7th, 2021. See the published version at https://doi.org/10.1186/s13643-021-01678-6. 


\title{
Deciphering the role of physical activity in stress management during a global pandemic in older adult populations:
}

\author{
a systematic review protocol
}

Authors:

${ }^{*}$ Corresponding author [(first) followed by remaining authors]

Mr. Ryan Churchill, Simon Fraser University, Department of Gerontology, ryan_churchill@sfu.ca

Mrs. Indira Riadi, Simon Fraser University, Department of Gerontology, indira_riadi@sfu.ca

Ms. Lucy Kervin, Simon Fraser University, Department of Gerontology, lucy_kervin@sfu.ca

Ms. Kelly Teo, Simon Fraser University, Department of Gerontology, kelly_teo@sfu.ca

Dr. Theodore Cosco, Simon Fraser University, Department of Gerontology, theodore_cosco@sfu.ca 


\section{Abstract \\ Background:}

The world has changed dramatically since the beginning of 2020 due to COVID-19. As a result of the pandemic, many older adults are now experiencing an increased and unprecedented amount of psychological stress. Physical activity has been found to be an evidence-based means of combating stress among older adults to promote their quality of life. Studies have demonstrated that those who are physically active experience fewer issues in regard to their mental health, specifically depression and anxiety disorders. Engagement in physical activity may exert a protective influence over stress inducing events and subsequent mental health outcomes. Due to the fact that exercise is inexpensive, non-invasive, and effective even via incremental increases in activity level, physical activity interventions should be investigated as an important therapy for reducing stress for older adults during this current pandemic.

\section{Methods:}

Four electronic databases will be searched to identify all randomized control trials that involve physical activity as an intervention to address stress 
management in older adults 50 years of age or older. Only peer reviewed and published journal articles will be reviewed. All studies will be assessed for bias using Cochrane's risk of bias tool. A random effects meta-analysis will be investigated if sufficient evidence of homogenous research exists and the heterogeneity of effect sizes will be tabulated.

\section{Discussion:}

This review will determine the effectiveness of various physical activity interventions for the treatment of stress among the older adult population. This knowledge will help inform care aides, clinicians, family members, and older adults themselves of the most effective physical activity interventions in dealing with stress which is relevant to the ongoing pandemic.

\section{Registration:}

PROSPERO CRD42020192546

\section{Keywords:}

Stress management, physical activity, pandemic, intervention, older adults, systematic review 


\section{Background}

The world as we know it has changed dramatically since the beginning of 2020, largely impacted by the pandemic caused by the SARS-COV-2 or more widely known as COVID-19. It has had a devastating effect not only with regards to global economies and health care outcomes (i.e. sickness and loss of life) but more specifically the older adult population worldwide [1]. According to the World Health Organization, older adults have an increased risk of developing serious symptoms from the illness [2]. At the onset of the pandemic, multiple stay at home orders were instituted. This prevented meaningful social connections and reduced possibilities of outdoor physical activity. When stuck at home in the middle of a pandemic due to a novel virus, older adults may turn to cable news for information. Before the pandemic, adults aged 65 and older, were the highest consumer of cable news [3]. Increase in television viewing time is associated with negative psychological and physiological manifestations [4]. Due to the uncertainty around the pandemic and the increase in time spent indoors, this potential for stress would only be exacerbated. Due to these factors and the increased 
covid-mortality as it relates to age [1], stress management in the senior population is of utmost importance for the foreseeable future.

When this pandemic began, older adults were subjected to a novel stress-invoking situation. Guided by Lazarus and Folkman's transactional theory of stress and coping [5], stress is defined as an imbalance between demands (environmental or intrapsychic) and an individual's available resources. Stress can occur when the pressure of an event or situation, such as the pandemic, surpasses a person's capabilities to cope. It is the interpretation of the event rather than the event itself that requires investigation. If the older adult is not able to cope with the stress, a litany of issues can result.

Researchers have found that cognitive interference by way of stress had a significant negative impact on cognitive performance (i.e. processing speed, episodic memory, and working memory) in later life [6]. A more dire result of unaddressed stress is suicide. During the SARS epidemic of 2003, there was a substantial spike (32\% increase ytd) in suicides for the subgroup of adults aged 65+ in Hong Kong [7]. In an important study looking at the suicide motives of older adults during this time, social disengagement, mental stress, anxiety, and the feeling of being a burden to their families were cited as reasons for the completed suicides [8]. More importantly for the current 
pandemic, they recommended that when developing epidemic control measures, that the mental and psychological wellbeing be taken into careful consideration, especially that of older adults.

One possible cost-effective, non-invasive intervention available to older adults to cope with these circumstances is physical activity. Studies have pointed to physically active people having reduced mental health problems, specifically depressive and anxiety disorders [9]. This evidence points to the wide ranging benefits of physical activity which will benefit many aspects of older adults' lives. Exercise has been shown to have a protective influence on mental health, specifically in alleviating stress, through the development of resilience. In one large study - The Copenhagen City Heart Study - using estimated activity, when physical activity was increased, there was a corresponding decrease in high levels of stress as well as a decrease in life dissatisfaction [10]. This study as well as Dunn's et al. [11] show the varying degrees of physical activity intensity in determining mood change (depression and anxiety). This is a key factor for consideration when comparing possible intervention strategies for older adults in that the largest increase in positive effects were seen in the comparison of sedentary individuals and light physical activity (2-4 hours per week). The individual does not have to be doing workouts at moderate to 
vigorous levels daily; gradually increasing baseline activity can be beneficial as well. In another study showing evidence of stress relief as it relates to being active, cancer patients, who were more active, were on average more resilient in general and had lower levels of stress. This effect increased with age [12].

In order to develop effective interventions to combat the current effects of stress, it is necessary to identify and understand the optimal type, intensity, and duration of the physical programs. Therefore, the objective of this systematic review is to examine the available evidence in order to identify the most effective and feasible physical activity interventions for older adults experiencing stress, particularly as it related to the current pandemic.

\section{Methods/Design}

This systematic review protocol has been registered with the International Prospective Register of Systematic Reviews (PROSPERO; Protocol ID: CRD42020192546). Any protocol amendments will be tracked. The Preferred Reporting Items for Systematic Review and Meta-Analysis Protocols 2015 checklist (PRISMA-P) was used to develop this protocol [13] (see Additional file 1).

Types of studies 
Experimental (randomized controlled trial (RCT)) study designs that compare acute or chronic stress before and after a physical activity intervention. Only original peer-reviewed published research will be included. No recent cutoff will be used in the search. Only studies written in English will be reviewed.

Types of participants

Studies including older adults of any gender without severe pre-existing medical conditions that may affect their ability to perform the intervention (e.g., coronary heart disease, severe cognitive impairment, etc.). Participants must be aged $\geq 50$ years.

Types of interventions

This review will include physical activity interventions that include aerobic and/or anaerobic activity and can include individual or group-sized interventions. Due to the variability of physical activity interventions, it is important that the study in question addresses the intensity - using the Copeland Threshold [14] (which has been developed for use with older adults) to distinguish between sedentary, low, moderate or vigorous physical activity and duration of the intervention used. Studies that do not include measurable physical activity outcomes will be excluded. 
Types of comparators

Comparator conditions will include participation in (a) a non-active activity or

(b) no intervention.

Types of outcomes

Physical activity must only be assessed using objective methods (e.g., pedometer, accelerometer, HR monitor, Vo2 Max, etc.). As it relates to stress, questionnaires, or survey data may be used to measure how stressful the individual perceives their current situation to be.

Search methods for the identification of studies

Data collection and analyses

The following databases will be searched: PubMed, Web of Science, PsycINFO, and SPORTDiscus.

The search will be restricted to studies published in English. Keywords will be related to stress management, older adults, and physical activity interventions. Appropriate keywords to identify studies using an experimental or randomized control study design will be employed. One example would be: 
"Physical activity intervention" AND "Stress" AND “Older Adults" OR "Seniors" OR "Elderly". For a study to be included it must include an appropriate control group. References of the included studies will be searched to identify additional potentially relevant studies.

Selection of studies

Articles will be imported into Microsoft Excel and all duplicates will be removed. Titles and abstracts will be screened for potential relevance. Full text of the relevant studies identified during previous screening will be reviewed to insure screening inclusion criteria was met. This screening will be done by two reviewers. Any disagreements will be resolved through discussion and consultation with a third reviewer.

Data extraction

This systematic review will conform to the guidelines outlined by the Preferred Reporting Items for Systematic Reviews and Meta-Analyses (PRISMA) checklist [12].

Extracted data will include information on publication (title, authors, year of publication, country of publication), population (participant sociodemographic characteristics, initial and final sample size), intervention 
type and dosage, control type and dosage, mode of intervention delivery (community centre, laboratory, other setting), type of analysis, outcomes (perceived stress via survey or questionnaire, heart rate, step count, oxygen levels, time intervals, BMI), dropouts, and any adverse effects. Specific characteristics will be collated into a table for the complete review.

Assessment of risk of bias in included studies

Risk of bias (ROB) assessment will be appropriately selected depending on the design of the studies included in the final synthesis. ROB will be evaluated both within and across included studies using Cochrane's risk of bias tool [15]. ROB will be assessed by 2 review authors, with discrepancies resolved by consensus with a third reviewer.

Data synthesis

A formal narrative synthesis is planned and studies that are included will be presented in summary tables with extracted data. Meta-analyses will only be performed when at least 3 included studies are sufficiently homogeneous in terms of study design, participants, interventions and outcomes to provide meaningful summary measures. Effect sizes expressed as odds ratios (for categorical data) and weighted mean differences (for continuous data) and 
95\% confidence intervals will be used for analysis. A random-effects meta-analysis will be performed due to the likelihood of similar effect sizes, but not completely uniform throughout all studies. Heterogeneity will be assessed using the $\mathrm{I}^{2}$ statistic with values above $75 \%$ and $\mathrm{p}<0.05$ used to indicate high heterogeneity [16]. If there is high heterogeneity, a meta-analysis will not be initiated. Where a meta-analysis is not possible, a narrative synthesis will be administered. The quality of the evidence will be assessed using the GRADE (Grading of Recommendations Assessment, Development and Evaluations) approach [17]. Finally, publication bias will be investigated by charting and deciphering the symmetry of a funnel plot for all studies considered in this review [18].

Subgroup analyses

If possible, subgroup analysis will be done to investigate if gender, BMI, or other factors play a role in stress level post intervention.

\section{Discussion}

This systematic review will be performed to compare the effectiveness of physical activity interventions for the promotion of stress management in older adults aged 50 years and older with non-active groups. Physical activity 
interventions should be scrutinized given the disparity between perceived physical fitness and health when compared to objective measurements[19]. This review is important because of the global attention on older adult demographics as it relates to the current pandemic. Physical activity interventions are advantageous in that they are easily accessible, can be performed with very little setup, and that most populations can participate in some fashion. Once these interventions are developed, adequately tested and tailored to individual needs of older adults, physical activity interventions can become a primary mechanism for stress management. Physical activity is both readily available and highly effective. Mounting evidence shows that physical activity is effective in reducing stress and its associated symptoms (heightened cortisol and increased heart rate) both independently and when compared to other techniques such as mindfulness [20]. Women who exercise consistently have been shown to have lower cortisol levels and heart rate in response to psychosocial stressors [21]. In another recent study by Garber [22], when looking at various defense mechanisms used to deal with pharmacy school stress, university students who used physical activity had significantly lower levels of perceived stress. While each student's perceived stress would differ, 
physical activity had the desired effect in reducing these symptoms and thus the students had a direct part in managing their stress.

Another important byproduct of physical activity as an effective stress management tool is the personal agency it gives older adults in actively dealing with stress. In a large university student sample, research provided evidence that individuals with an internal locus of control consistently had decreased levels of anxiety and increased problem solving coping skills when compared to those with an external locus of control [23]. Realizing that they can actively reduce their own stress by shifting or keeping their locus of control internally can be an empowering moment for older adults during the current pandemic.

To our knowledge, this will be the first review that synthesizes information on the effectiveness of physical activity interventions promoting stress management in older adults aged 50 years and above. We also hope to find evidence of the most effective type of physical activity given the likelihood of variability in the type, duration, and quality of effective interventions. This will increase efficiency and effectiveness among health practitioners as well as older adults themselves as it relates to the timely treatment of stress. 
The results of this review will provide evidence to the role of physical activity in a national, governmental, stress management guideline for older adults dealing with stress. Findings can also provide care aides, hospital staff, and/or family members with evidence on how best to help the older adult. Ultimately, this systematic review should provide evidence for non-pharmacological, effective, physical activity treatments that aim to limit the potentially debilitating role of stress in the older adult population.

\section{Abbreviations}

CDC - Center for Disease Control and Prevention

HR - Heart Rate

PRISMA-P - Preferred Reporting Items for Systematic Reviews and Meta-Analyses Protocols

PROSPERO: International Prospective Register of Systematic Reviews

ROB - Risk of Bias

VO2 Max - Maximum Oxygen Volume

WHO - World Health Organization 


\section{Declarations}

Ethics approval and consent to participate

Not applicable

Consent for publication

Not applicable

Availability of supporting data

Not applicable

Competing interests

The authors declare that they have no competing interests.

\section{Funding}

Not applicable

\section{Authors' Contributions}

$\mathrm{RC}$ is the guarantor of the review protocol, designed the protocol, and drafted the manuscript. RC will be the first reviewer for this systematic review. IR, LK, 
and KT provided guidance and contributed to editing sections of the manuscript. TC was the senior researcher on the team. TC also provided key insight into edits and conceptualization of the protocol. All authors revised and approved the final version of the manuscript.

Acknowledgments

Not Applicable

Authors' Information

Affiliations

1. School of Gerontology, Simon Fraser University, Vancouver, V6B $5 \mathbf{K} 3$

Ryan Churchill, Indira Riadi, Lucy Kervin, Kelly Teo \& Theodore Cosco

Corresponding author

Ryan Churchill 


\section{References}

1. Covid CD, Team R. Severe outcomes among patients with coronavirus disease 2019 (COVID-19)-United States, February 12-March 16, 2020. MMWR Morb Mortal Wkly Rep. 2020;69(12):343-6.

2. World Health Organization. Coronavirus URL:

https://www.who.int/health-topics/coronavirus\#tab=tab 1 [accessed 2020-06-24]

3. Kohut A, Doherty C, Dimock M, Keeter S. In changing news landscape, even television is vulnerable. Pew Internet \& American Life Project. 2012 Sep 27.

4. Hamer M, Poole L, Messerli-Bürgy N. Television viewing, C-reactive protein, and depressive symptoms in older adults. BRAIN BEHAV IMMUN. 2013 Oct 1;33:29-32.

5. Lazarus RS, Folkman S. Stress, appraisal, and coping. Springer publishing company; 1984 Mar 15. 
6. Stawski RS, Sliwinski MJ, Smyth JM. Stress-related cognitive interference predicts cognitive function in old age. Psychol. Aging. 2006 Sep;21(3):535.

7. Cheung YT, Chau PH, Yip PS. A revisit on older adults suicides and Severe Acute Respiratory Syndrome (SARS) epidemic in Hong Kong. Int. J. Geriatr. Psychiatry. 2008 Dec;23(12):1231-8.

8. Yip PS, Cheung YT, Chau PH, Law YW. The impact of epidemic outbreak: the case of severe acute respiratory syndrome (SARS) and suicide among older adults in Hong Kong. Crisis: Crisis. 2010;31(2):86.

9. Goodwin RD. Association between physical activity and mental disorders among adults in the United States. Prev. Med. 2003 Jun 1;36(6):698-703.

10. Schnohr P, Kristensen TS, Prescott E, Scharling H. Stress and life dissatisfaction are inversely associated with jogging and other types of physical activity in leisure time-The Copenhagen City Heart Study. Scand. J. Med. Sci. Sports. 2005 Apr;15(2):107-12. 
11. Dunn AL, Trivedi MH, O’Neal HA. Physical activity dose-response effects on outcomes of depression and anxiety. Copenhagen City Heart Study 111 Med Sci Sports Exer. 2001: 33: S587-S597.

12. Matzka M, Mayer H, Köck-Hódi S, Moses-Passini C, Dubey C, Jahn P, Schneeweiss S, Eicher M. Relationship between resilience, psychological distress and physical activity in cancer patients: A cross-sectional observation study. PloS one. 2016 Apr 28;11(4):e0154496.

13. Moher D, Shamseer L, Clarke M, Ghersi D, Liberati A, Petticrew M, Shekelle P, Stewart LA. Preferred reporting items for systematic review and meta-analysis protocols (PRISMA-P) 2015 statement. Syst. Rev. 2015 Dec $1 ; 4(1): 1$.

14. Copeland JL, Esliger DW. Accelerometer assessment of physical activity in active, healthy older adults. J AGING PHYS ACTIV. 2009 Jan 1;17(1):17-30.

15. Higgins JP, Thomas J, Chandler J, Cumpston M, Li T, Page MJ, Welch VA, editors. Cochrane handbook for systematic reviews of interventions. John Wiley \& Sons; 2019 Sep 23. 
16. Higgins JP, Thompson SG, Deeks JJ, Altman DG. Measuring inconsistency in meta-analyses. BMJ. 2003 Sep 4;327(7414):557-60.

17. Guyatt G, Oxman AD, Akl EA, Kunz R, Vist G, Brozek J, Norris S, Falck-Ytter Y, Glasziou P, Debeer H, Jaeschke R. GRADE guidelines: 1. Introduction-GRADE evidence profiles and summary of findings tables. J. Clin. Epidemiol. 2011 Apr 1;64(4):383-94.

18. Sterne JA, Sutton AJ, Ioannidis JP, Terrin N, Jones DR, Lau J, Carpenter J, Rücker G, Harbord RM, Schmid CH, Tetzlaff J. Recommendations for examining and interpreting funnel plot asymmetry in meta-analyses of randomised controlled trials. BMJ. $2011 \mathrm{Jul} \mathrm{22;343.}$

19. Wells EK, Avery ML, Eschbach LC, Bunn J. A Comparison of Perceived Physical Fitness and Objective Measurements. J. Sports Sci. 2016 Oct 1.

20. Pinniger R, Brown RF, Thorsteinsson EB, McKinley P. Argentine tango dance compared to mindfulness meditation and a waiting-list control: A randomised trial for treating depression. COMPLEMENT THER MED. 2012 Dec 1;20(6):377-84.

21. Klaperski S, von Dawans B, Heinrichs M, Fuchs R. Does the level of physical exercise affect physiological and psychological responses to 
psychosocial stress in women?. J Sport Exerc Psychol. 2013 Mar 1;14(2):266-74.

22. Garber MC. Exercise as a stress coping mechanism in a pharmacy student population. Am. J. Pharm. Educ. 2017 Apr 1;81(3).

23. Arslan C, Dilmaç B, Hamarta E. Coping with stress and trait anxiety in terms of locus of control: A study with Turkish university students. Soc.

Behav. Pers. 2009 Jul 1;37(6):791-80o. 


\section{Supplementary Files}

This is a list of supplementary files associated with this preprint. Click to download.

- PRISMAPchecklist.docx 